$D$-tartaric acids, glycine and the sodium salt of $\mathrm{L}-\alpha$-hydroxy- $\beta$-sulphopropionic acid.

2. Thiocyanate and the sodium salt of acetylenedicarboxylic acid were found to be non-competitive inhibitors.

3. The following compounds were found to be without effect on fumarase: the sodium salts of acetic, butyric, crotonic, $\mathrm{L}$ - and DL-aspartic, and acetoacetic acids, the mono- and di-methyl esters of fumarate, and the SH inhibitors, iodoacetate, iodoacetamide, iodosobenzoate, $p$-chloromercuribenzoate and chloroacetophenone.

4. The inhibition of all the competitive inhibitors was dependent on the temperature. Of the three inhibitors investigated in detail at low temperatures the combination of fumarase and inhibitors was found to be exothermic, while at higher temperatures the combination was found to be endothermic.
5. Inhibition by all the competitive inhibitors tested was markedly dependent on $\mathrm{pH}$. The variation of the inhibitor constants and of the Michaelis constants with $\mathrm{pH}$ has been studied in detail, and the results are discussed in the light of the theory of Dixon (1953).

6. A tentative hypothesis of the structure of the active centre and the sequence of reactions which may accompany the reversible hydration of fumarate to L-malate is suggested on the basis of these results.

I am indebted to Dr M. Dixon, F.R.S., for his helpful advice and for his interest in this work. I also wish to thank him for information about his enzyme-substrate theory before publication. I am also grateful to other members of the department, in particular Dr G. Weber and Dr E. C. Webb for many helpful discussions. This work was carried out on a grant from the 'Commonwealth Scientific and Industrial Research Organization, Australia.

\title{
REFERENCES
}

Dixon, M. (1950). Multi-enzyme Systems. Cambridge Lineweaver, H. \& Burk, D. (1934). J. Amer. chem. Soc. 56, University Press.

Dixon, M. (1953). Biochem. J. 55, 161.

Laki, K. (1941). Enzymologia, 9, 141.

658.

Massey, V. (1953a). Biochem. J. 53, 67.

Massey, V. (1953b). Biochem. J. 53, 72.

\section{Absorption of Immune Globulin by the Young Lamb after Ingestion of Colostrum}

\author{
By E. F. McCARTHY AND E. I. McDOUGALL \\ The Lister Institute, London, * and the Rowett Research Institute, \\ Bucksburn, Aberdeenshire
}

(Received 26 November 1952)

The role of colostrum in promoting the well being of the young ruminant has been established mainly by work on the cow. It has been shown that these animals depend on the ingestion of colostrum soon after birth and the absorption of immune globulins from this secretion for a passive immunity which greatly favours their chances of survival of the critical first fow weeks of their life (Smith \& Little, 1922; Orcutt \& Howe, 1922). That a similar situation obtains in the sheep has been shown in three different ways; the immunological studies of Mason, Dalling \& Gordon (1930) and Schnieder \& Szathmary (1939); the work on the sodium sulphate salted-out protein fractions of Earle (1935) and the electrophoretic examinations of Charlwood \& Thomson (1948). In the present work these three methods have been combined in a study of the changes in the serum proteins of suckling lambs and

* Present address. Physiology Department, University College, Dublin. extended to a series of lambs receiving colostrum after various periods of delay. In relation to these changes, the existence and nature of any proteinuria in the young lambs has been investigated.

\section{METHODS •}

Salted-out nitrogen fractions. Serum protein, globulin and albumin $\mathrm{N}$ fractions were estimated by difference from the total N, 'globulin-free' $N$ and non-protein $N$ contents; these were determined by micro-Kjeldahl analysis of the following solutions representing a dilution of 1 in 21 of the serum sample: a dilution in normal saline, a globulin-free filtrate obtained after precipitation with saturated $\mathrm{MgSO}_{4}$ at $20^{\circ}$ overnight, and a protein-free filtrate obtained after precipitation with trichloroacetic acid (5 g./100 ml.). Colostrum $\mathrm{N}$ fractions were obtained by analysing a suitable dilution in saline in the same way as serum with the following addition: casein $\mathbf{N}$ was obtained by including the analysis of a caseinfree filtrate prepared by acidification of a dilution in saline with acetic acid. 
Electrophoretic examination of the sera. This was carried out in the Tiselius apparatus at $4^{\circ}$ using phosphate buffer at $\mathrm{pH} 8.0$ and ionic strength 0.2 and a protein concentration of about $2 \mathrm{~g} . / 100 \mathrm{ml}$. The percentage composition was determined from the diagrams by projection, extrapolation and planimetry. As considerable difficulty was experienced in analysing the individual components, the method of extrapolation employed by Dalgarno, Godden \& McCarthy (1950) was used throughout. The albumin and salt boundaries were completed. The slowest component, probably corresponding to the $\gamma$-globulin of human serum was then extrapolated and called component 2 . The area between this component and the albumin was not subjected to further analysis but was measured as a single component and termed component 1 .

Antibodies. Antibodies in colostrum, serum and urine were estimated by the limiting dilution of the agglutinin test. At first the tests were carried out from a lower limit of 1 in 25 , but later, samples which were negative at this limit were examined on a lower range from a dilution of 1 in 2 .

Urine examination. This consisted of qualitative tests for protein by sulphosalicylic acid precipitation and by heat coagulation (with addition of acetic acid). Globulin was detected by precipitation with half-saturated $\left(\mathrm{NH}_{4}\right)_{2} \mathrm{SO}_{4}$ and with saturated $\mathrm{MgSO}_{4}$. These tests were made comparative by an arbitrary grading of the degree of precipitation as a trace,,+++ and +++ . Antibodies were tested for in the lower range of dilutions, that is from a dilution of 1 in 2.

\section{RESULTS}

The present observations were made on Cheviot, Blackface and half-bred lambs over a period of two lambing seasons. The ewes which gave birth to them were immunized with Salmonella typhosa antigen. The first injection was given 21 days before the expected lambing date. After birth, some lambs were allowed to suck the ewe in the normal way, some were bottle-fed with cows' milk for varying periods before colostrum feeding, while others never received colostrum. The lambs which received colostrum after a delay, did so, either by returning to the unsucked dam when the delay was short, or by being fostered on to another ewe which had recently lambed but not suckled, or by ingesting a single pint of stored colostrum. The relations and treatments of these animals are given in Table 1.

Observations were made on 15 ewes and 17 lambs. Seven lambs ingested colostrum at birth, six after delays of 12, 24, 29 and $48 \mathrm{hr}$. and 3 and 9 days, respectively, and four were deprived of colostrum.

The serum and colostrum of the ewes at lambing were examined for salted-out nitrogen fractions and antibodies. In two ewes, the colostrum was further examined at various intervals after lambing. In two other ewes it was also examined before lambing took place. The results presented for the ewes, Table 2, have been limited to thirteen relating to those given for the lambs, Table 3.

The lambs' serum was also examined by these two methods and in addition by electrophoretic analysis. Samples were taken at the following times: birth, 2-3 days, 7-10 days and at 5-6 weeks after birth. The results, Table 3 , in the case of lambs ingesting colostrum at birth, have been limited to four typical cases. The urine of twelve lambs was examined for a few days after birth and ingestion of colostrum, Table 4.

The composition of colostrum, Table 2, showed at lambing protein nitrogen ranging from approximately 1 to $4 \mathrm{~g}$. $/ 100 \mathrm{ml}$. and titres from 1 in 1000 to 1 in more than 10000 . The subsequent rapid change in composition is illustrated by the data included for ewes 232 and 255, Table 2.

The observations on colostrum before lambing were made on ewes 13 and 22, Table 2 . The colostrum of ewe 13 obtained 3 days before lambing resembled the richer samples in its nitrogen distribution and agglutinin titre. The other, from ewe 22, 12 days

Table 1. Lambs, dams and treatments

\begin{tabular}{|c|c|c|}
\hline \multicolumn{3}{|c|}{ Kept on the ewe } \\
\hline Lamb no. & Ewe no. & Breed \\
\hline 512 & 178 & Cheviot \\
\hline $518^{\circ}$ & 191 & Cheviot \\
\hline 672 & 46 & Half-bred \\
\hline 673 & 42 & Half-bred \\
\hline 675 & 28 & Half-bred \\
\hline 678 & 51 & Half-bred \\
\hline 686 & 22 & Half-bred \\
\hline
\end{tabular}

\begin{tabular}{ccl}
\multicolumn{3}{c}{ No colostrum } \\
Lamb no. & Ewe no. & Breed \\
452 & $255^{*}$ & Blackface \\
415 & 232 & Blackface \\
514 & 190 & Cheviot \\
674 & 28 & Half-bred
\end{tabular}

Colostrum delayed

\begin{tabular}{crlll}
\hline Lamb no. & Ewe no. & Breed & Delay & Method of receiving colostrum \\
680 & 53 & Half-bred & $12 \mathrm{hr}$. & Returned to dam \\
683 & 13 & Half-bred & $24 \mathrm{hr}$. & Returned to dam \\
682 & 26 & Half-bred & $29 \mathrm{hr}$. & Given 200 ml. colostrum from ewe 53 \\
519 & 191 & Cheviot & $48 \mathrm{hr}$. & Fostered on to ewe 172 \\
677 & 51 & Half-bred & 3 days & Fostered on to ewe 37 \\
513 & 178 & Cheviot & 9 days & Fostered on to ewe 190
\end{tabular}


Table 2. Nitrogen distribution and titres of ewes' serum and colostrum

\begin{tabular}{|c|c|c|c|c|c|c|c|c|c|c|}
\hline \multirow{3}{*}{$\begin{array}{l}\text { Ewe } \\
\text { no. }\end{array}$} & \multirow{3}{*}{$\begin{array}{l}\text { Days from } \\
\text { lambing }\end{array}$} & \multicolumn{4}{|c|}{ Serum } & \multicolumn{5}{|c|}{ Colostrum } \\
\hline & & \multicolumn{3}{|c|}{$\mathrm{N} \mathrm{mg./100} \mathrm{ml.}$} & \multirow[b]{2}{*}{ Titre } & \multicolumn{4}{|c|}{$\mathrm{N} \mathrm{mg./100} \mathrm{ml.}$} & \multirow[b]{2}{*}{ Titre } \\
\hline & & Protein & Globulin & Albumin & & Protein & Casein & Globulin & Albumin & \\
\hline 13 & $\begin{array}{l}3 \text { before } \\
0 \text { after }\end{array}$ & $\overline{1251}$ & $\overline{590}$ & $\overline{661}$ & $\overline{500}$ & $\begin{array}{l}3767 \\
2635\end{array}$ & $\begin{array}{l}645 \\
813\end{array}$ & $\begin{array}{l}2732 \\
1347\end{array}$ & $\begin{array}{l}390 \\
475\end{array}$ & $\begin{array}{r}7500 \\
>10000\end{array}$ \\
\hline 22 & $\begin{array}{l}12 \text { before } \\
0 \text { after }\end{array}$ & $\overline{1095}$ & $\overline{589}$ & $\overline{506}$ & $\overline{500}$ & $\begin{array}{l}5275 \\
2706\end{array}$ & $\begin{array}{l}755 \\
210\end{array}$ & $\begin{array}{l}4045 \\
2157\end{array}$ & $\begin{array}{l}475 \\
339\end{array}$ & $\begin{array}{r}<25 \\
1000\end{array}$ \\
\hline $\begin{array}{r}26 \\
28 \\
37 \\
51 \\
53 \\
172 \\
178 \\
190 \\
191\end{array}$ & 0 after & $\begin{array}{r}1082 \\
1036 \\
1241 \\
1015 \\
1170 \\
900 \\
982 \\
928 \\
900\end{array}$ & $\begin{array}{l}568 \\
555 \\
725 \\
573 \\
670 \\
430 \\
486 \\
442 \\
430\end{array}$ & $\begin{array}{l}514 \\
481 \\
516 \\
442 \\
500 \\
470 \\
496 \\
486 \\
470\end{array}$ & $\begin{array}{r}2500 \\
500 \\
2500 \\
10000 \\
1000 \\
1000 \\
2500 \\
5000 \\
1000\end{array}$ & $\begin{array}{l}4136 \\
3390 \\
1183 \\
2047 \\
2174 \\
3100 \\
2980 \\
2990 \\
2677\end{array}$ & $\begin{array}{r}1122 \\
1390 \\
613 \\
879 \\
875 \\
1245 \\
804 \\
1410 \\
1357\end{array}$ & $\begin{array}{r}2288 \\
1565 \\
300 \\
838 \\
1096 \\
1317 \\
1817 \\
1186 \\
955\end{array}$ & $\begin{array}{l}726 \\
\mathbf{4 3 5} \\
270 \\
\mathbf{3 3 0} \\
\mathbf{2 0 3} \\
\mathbf{5 3 8} \\
\mathbf{3 5 9} \\
\mathbf{3 9 4} \\
\mathbf{3 6 5}\end{array}$ & $\begin{array}{r}2500 \\
1000 \\
1000 \\
2500 \\
2500 \\
2500 \\
>10000 \\
>10000 \\
2500\end{array}$ \\
\hline 232 & $\begin{array}{r}0 \text { after } \\
3 \text { after } \\
9 \text { after } \\
36 \text { after }\end{array}$ & $\frac{1046}{\overline{1213}}$ & $\frac{442}{568}$ & $\frac{604}{-}$ & $\frac{5000}{-}$ & $\begin{array}{r}2956 \\
1126 \\
697 \\
687\end{array}$ & $\begin{array}{r}1070 \\
734 \\
588 \\
566\end{array}$ & $\begin{array}{r}1473 \\
253 \\
41 \\
56\end{array}$ & $\begin{array}{r}413 \\
139 \\
68 \\
65\end{array}$ & $\begin{array}{r}10000 \\
2500 \\
250 \\
<25\end{array}$ \\
\hline 255 & $\begin{array}{r}0 \text { after } \\
2 \text { after } \\
7 \text { after } \\
40 \text { after }\end{array}$ & $\frac{904}{\overline{1242}}$ & $\frac{354}{-}$ & $\frac{550}{-}$ & $\begin{array}{l}- \\
-\end{array}$ & $\begin{array}{r}3742 \\
1191 \\
786 \\
859\end{array}$ & $\begin{array}{l}730 \\
756 \\
629 \\
696\end{array}$ & $\begin{array}{r}2445 \\
277 \\
66 \\
63\end{array}$ & $\begin{array}{r}567 \\
158 \\
91 \\
100\end{array}$ & $\overline{-}$ \\
\hline
\end{tabular}

before lambing was most concentrated containing $5 \mathrm{~g} . / 100 \mathrm{ml}$. protein nitrogen of which $77 \%$ was globulin nitrogen but its titre was less than 1 in 25 . However, the colostrum from the same ewe at lambing had a titre of 1 in 1000. This suggests that the antibodies may be secreted in the colostrum over a relatively short period immediately before lambing. Mason et al. (1930) found antitoxin to lamb dysentery present 12 days before lembing, but their ewes had been immunized throughout pregnancy.

The changes in the lambs' serum proteins. The changes shown in Table 3 and Fig. 1 illustrate the effects of the post-natal occurrence and subsequent cessation of colostrum absorption. The degree to which they do so varies with the method of analysis.

The analysis of globulin nitrogen by salt precipitation showed a marked temporary increase in both absolute and relative concentrations after ingestion of colostrum, when this took place at birth or after $12 \mathrm{hr}$. delay, inconclusive changes when the delay was 24,29 and $48 \mathrm{hr}$. and a gradual increase in absolute but not relative concentration over the period of observation when the delay was longer or when no colostrum was given.

The electrophoretic results showed similar trends in protein composition, but in addition an increase in the proportion of globulin when the delay was $24 \mathrm{hr}$. The approximate average values of the relative globulin concentrations were $21 \%$ at birth, $55 \%$ a few days after absorption of colostrum and $33 \% 5$ or 6 weeks later. These changes were mainly due to the globulin referred to as component 2. Its corresponding values were 5, 31 and $11 \%$ respectively. Component 1 remained at about $22 \%$ throughout. By the time the lambs were 5 or 6 weeks old there was little difference in their serum globulin determined by either method whether they had shown absorption or not.

The agglutinin titre clearly showed absorption of globulin in the lambs receiving colostrum up to $29 \mathrm{hr}$. but not after $48 \mathrm{hr}$. or more delay. The titres of animals showing absorption rose from negligible values at birth to between 1 in 125 and 1 in 5000 after ingestion of colostrum and fell to quite low values 5 or 6 weeks later.

Comparison of the results obtained on the serum of lambs 512, 518, 678 and 686 (Table 3) with those obtained on the corresponding ewes, 178, 191, 51 and 22 respectively (Table 2) shows that colostrum ingestion and absorption had given to the lamb for the time being a globulin-nitrogen content and an agglutination titre comparable with that of its dam. The lambs serum taken at varying periods after birth is compared with that of the dam at birth only since data on these ewes' serum at corresponding post-natal periods was not obtained. 5 or 6 weeks later the lambs' globulin nitrogen was much less and its titre, as expected, quite small compared with its dam. During this period the lambs' albumin nitrogen rose to a level comparable to that in the dam.

The relative globulin concentration of the lambs' serum has been calculated from the salt precipitation results and included in Table 3. Comparison with the electrophoretically determined globulin shows that they have the same general trends but 
Table 3. Examination of lambs' serum

\begin{tabular}{|c|c|c|c|c|c|c|c|c|c|c|c|}
\hline \multirow{3}{*}{$\begin{array}{c}\text { Lamb } \\
\text { no. }\end{array}$} & \multirow[b]{3}{*}{ Delay } & \multirow{3}{*}{$\begin{array}{c}\text { Age } \\
\text { (days) }\end{array}$} & \multirow{2}{*}{\multicolumn{3}{|c|}{$\begin{array}{l}\text { Nitrogen distribution } \\
\quad(\mathrm{mg} . / 100 \mathrm{ml} .)\end{array}$}} & \multirow[b]{3}{*}{ Titre } & \multirow[b]{3}{*}{ Albumin } & \multicolumn{3}{|c|}{ By electrophoresis } & \multirow{3}{*}{$\begin{array}{l}\text { By MgSC } \\
\text { pptn. } \\
\text { globulin }\end{array}$} \\
\hline & & & & & & & & \multirow{2}{*}{$\begin{array}{c}\text { Com- } \\
\text { ponent } \\
1\end{array}$} & \multirow{2}{*}{$\begin{array}{l}\text { Com- } \\
\text { ponent } \\
\quad 2\end{array}$} & \multirow[b]{2}{*}{ Globulin } & \\
\hline & & & Protein & Globulin & Albumin & & & & & & \\
\hline \multicolumn{12}{|c|}{ Kept on the ewe } \\
\hline 512 & - & $\begin{array}{r}0 \\
2 \\
12 \\
33\end{array}$ & $\begin{array}{r}739 \\
785 \\
1030 \\
965\end{array}$ & $\begin{array}{l}270 \\
421 \\
492 \\
353\end{array}$ & $\begin{array}{l}469 \\
364 \\
538 \\
612\end{array}$ & $\begin{array}{r}<25 \\
2500 \\
2500 \\
500\end{array}$ & $\begin{array}{c}73 \cdot 8 \\
\mathbf{3 4 \cdot 6} \\
\mathbf{5 2 \cdot 3} \\
-\end{array}$ & $\begin{array}{l}22 \cdot 0 \\
22 \cdot 6 \\
27 \cdot 7 \\
-\end{array}$ & $\begin{array}{r}4 \cdot 2 \\
42 \cdot 8 \\
20 \cdot 1 \\
13 \cdot 5\end{array}$ & $\begin{array}{c}26 \cdot 2 \\
65 \cdot 4 \\
47 \cdot 7 \\
-\end{array}$ & $\begin{array}{l}\mathbf{3 7 \cdot 2} \\
\mathbf{5 4 \cdot 2} \\
\mathbf{4 8 \cdot 5} \\
\mathbf{3 7 \cdot 4}\end{array}$ \\
\hline 518 & - & $\begin{array}{r}2 \\
10 \\
36\end{array}$ & $\begin{array}{l}909 \\
936 \\
882\end{array}$ & $\begin{array}{l}476 \\
313 \\
248\end{array}$ & $\begin{array}{l}433 \\
623 \\
634\end{array}$ & $\begin{array}{r}1000 \\
250 \\
50\end{array}$ & $\begin{array}{l}49 \cdot 0 \\
53 \cdot 5 \\
63 \cdot 2\end{array}$ & $\begin{array}{l}23 \cdot 7 \\
28 \cdot 0 \\
20 \cdot 1\end{array}$ & $\begin{array}{l}27 \cdot 3 \\
18 \cdot 5 \\
16 \cdot 7\end{array}$ & $\begin{array}{l}51 \cdot 0 \\
46 \cdot 5 \\
36 \cdot 8\end{array}$ & $\begin{array}{l}53 \cdot 2 \\
34 \cdot 1 \\
28 \cdot 8\end{array}$ \\
\hline 678 & - & $\begin{array}{r}0 \\
4 \\
10 \\
33\end{array}$ & $\begin{array}{r}713 \\
953 \\
920 \\
1077\end{array}$ & $\begin{array}{l}269 \\
456 \\
352 \\
488\end{array}$ & $\begin{array}{l}\mathbf{4 4 4} \\
\mathbf{4 9 7} \\
\mathbf{5 6 8} \\
\mathbf{5 8 9}\end{array}$ & $\begin{array}{l}<2 \\
500 \\
125 \\
<25\end{array}$ & $\begin{array}{l}76 \cdot 3 \\
50 \cdot 7 \\
-\overline{68 \cdot 1}\end{array}$ & $\begin{array}{l}21 \cdot 1 \\
24 \cdot 9 \\
\overline{25 \cdot 3}\end{array}$ & $\begin{array}{c}2 \cdot 6 \\
24 \cdot 4 \\
\overline{6 \cdot 6}\end{array}$ & $\begin{array}{c}23 \cdot 7 \\
49 \cdot 3 \\
-\overline{31 \cdot 9}\end{array}$ & $\begin{array}{l}38 \cdot 3 \\
48 \cdot 7 \\
39 \cdot 2 \\
46 \cdot 0\end{array}$ \\
\hline 686 & - & $\begin{array}{l}2 \frac{1}{4} \\
10^{\frac{1}{2}} \\
51\end{array}$ & $\begin{array}{l}\frac{878}{982} \\
935\end{array}$ & $\begin{array}{l}\frac{481}{445} \\
294\end{array}$ & $\begin{array}{l}\frac{397}{537} \\
641\end{array}$ & $\begin{array}{r}250 \\
500 \\
1000 \\
<25\end{array}$ & $\begin{array}{c}53 \cdot 9 \\
35 \cdot 6 \\
51 \cdot 6 \\
-\end{array}$ & $\begin{array}{c}20.9 \\
23 \cdot 0 \\
24 \cdot 5 \\
-\end{array}$ & $\begin{array}{c}25 \cdot 2 \\
38 \cdot 4 \\
23 \cdot 9 \\
-\end{array}$ & $\begin{array}{c}46 \cdot 1 \\
61 \cdot 4 \\
48 \cdot \dot{4} \\
-\end{array}$ & $\begin{array}{l}55 \cdot 5 \\
-46 \cdot 2 \\
32 \cdot 2\end{array}$ \\
\hline \multicolumn{12}{|c|}{ Colostrum delayed } \\
\hline 680 & $12 \mathrm{hr}$. & $\begin{array}{r}\mathbf{0} \\
\mathbf{3} \\
10 \\
\mathbf{3 3}\end{array}$ & $\begin{array}{r}801 \\
1029 \\
1042 \\
847\end{array}$ & $\begin{array}{l}291 \\
542 \\
502 \\
315\end{array}$ & $\begin{array}{l}510 \\
487 \\
540 \\
532\end{array}$ & $\begin{array}{r}<2 \\
2500 \\
250 \\
125\end{array}$ & $\begin{array}{c}-\overline{40 \cdot 7} \\
51 \cdot 6 \\
74 \cdot 2\end{array}$ & $\begin{array}{l}\overline{24 \cdot 5} \\
25 \cdot 7 \\
14 \cdot 6\end{array}$ & $\begin{array}{l}-\overline{34 \cdot 8} \\
22 \cdot 7 \\
11 \cdot 2\end{array}$ & $\begin{array}{l}-\overline{59 \cdot 3} \\
48 \cdot 4 \\
25 \cdot 8\end{array}$ & $\begin{array}{l}37 \cdot 0 \\
53 \cdot 5 \\
49 \cdot 0 \\
38 \cdot 0\end{array}$ \\
\hline 683 & $24 \mathrm{hr}$. & $\begin{array}{l}3 \frac{1}{4} \\
3 \frac{1}{4} \\
29\end{array}$ & $\begin{array}{l}787 \\
893 \\
752 \\
762\end{array}$ & $\begin{array}{l}316 \\
367 \\
278 \\
270\end{array}$ & $\begin{array}{l}\mathbf{4 7 1} \\
526 \\
474 \\
492\end{array}$ & $\begin{array}{r}<2 \\
1000 \\
50 \\
50\end{array}$ & $\begin{array}{l}72 \cdot 8 \\
61 \cdot 9 \\
62 \cdot 2 \\
69 \cdot 1\end{array}$ & $\begin{array}{l}21 \cdot 1 \\
27 \cdot 0 \\
28 \cdot 0 \\
23 \cdot 3\end{array}$ & $\begin{array}{r}6 \cdot 1 \\
11 \cdot 1 \\
8 \cdot 8 \\
7 \cdot 6\end{array}$ & $\begin{array}{l}27 \cdot 2 \\
38 \cdot 1 \\
36 \cdot 8 \\
30 \cdot 9\end{array}$ & $\begin{array}{l}40 \cdot 8 \\
41 \cdot 8 \\
37 \cdot 7 \\
36 \cdot 2\end{array}$ \\
\hline 682 & $29 \mathrm{hr}$. & $\begin{array}{r}0 \\
10 \\
31\end{array}$ & $\begin{array}{l}717 \\
860 \\
812\end{array}$ & $\begin{array}{l}289 \\
380 \\
303\end{array}$ & $\begin{array}{l}428 \\
480 \\
509\end{array}$ & $\begin{array}{r}16 \\
1000 \\
125\end{array}$ & $\bar{z}$ & $\bar{z}$ & $\bar{z}$ & E & $\begin{array}{l}41 \cdot 2 \\
45 \cdot 0 \\
38 \cdot 0\end{array}$ \\
\hline 519 & $48 \mathrm{hr}$. & $\begin{array}{r}2 \\
5 \\
10 \\
36\end{array}$ & $\begin{array}{l}597 \\
715 \\
759 \\
929\end{array}$ & $\begin{array}{l}182 \\
265 \\
149 \\
344\end{array}$ & $\begin{array}{l}415 \\
450 \\
610 \\
585\end{array}$ & $\begin{array}{r}<2 \\
<25 \\
<2 \\
<2\end{array}$ & $\begin{array}{l}60 \cdot 4 \\
64 \cdot 9 \\
65 \cdot 9 \\
64 \cdot 3\end{array}$ & $\begin{array}{l}26 \cdot 7 \\
\overline{27 \cdot 4} \\
23 \cdot 4\end{array}$ & $\begin{array}{c}12 \cdot 9 \\
6 \cdot 7 \\
12 \cdot 3\end{array}$ & $\begin{array}{l}39 \cdot 6 \\
35 \cdot 1 \\
34 \cdot 1 \\
35 \cdot 7\end{array}$ & $\begin{array}{l}31 \cdot 2 \\
38 \cdot 0 \\
20 \cdot 0 \\
37 \cdot 7\end{array}$ \\
\hline 677 & 3 days & $\begin{array}{r}0 \\
3 \\
5 \\
10 \\
33\end{array}$ & $\begin{array}{l}677 \\
620 \\
792 \\
785\end{array}$ & $\begin{array}{l}235 \\
219 \\
249 \\
283\end{array}$ & $\begin{array}{l}442 \\
401 \\
543 \\
502\end{array}$ & $\begin{array}{l}<2 \\
<2 \\
<2 \\
<2 \\
<2\end{array}$ & $\begin{array}{c}\overline{71 \cdot 4} \\
\overline{68 \cdot 5} \\
71 \cdot 7\end{array}$ & $\begin{array}{l}\overline{23 \cdot 8} \\
\overline{23.4} \\
18.5\end{array}$ & $\begin{array}{l}6 \cdot 2 \\
4 \cdot 8 \\
8 \cdot 1 \\
9 \cdot 7\end{array}$ & $\begin{array}{l}\overline{28 \cdot 6} \\
\overline{31 \cdot 5} \\
28 \cdot 2\end{array}$ & $\begin{array}{l}35 \cdot 3 \\
\overline{36 \cdot 2} \\
32 \cdot 0 \\
36 \cdot 6\end{array}$ \\
\hline 513 & $9 \operatorname{davs}$ & $\begin{array}{r}0 \\
9 \\
12 \\
\mathbf{3 3}\end{array}$ & $\begin{array}{l}529 \\
757 \\
812 \\
792\end{array}$ & $\begin{array}{l}194 \\
262 \\
228 \\
241\end{array}$ & $\begin{array}{l}\mathbf{3 3 5} \\
\mathbf{4 9 5} \\
\mathbf{5 8 4} \\
\mathbf{5 5 1}\end{array}$ & $\begin{array}{l}<25 \\
<25 \\
<25 \\
<25\end{array}$ & $\begin{array}{l}-\overline{61 \cdot 4} \\
59 \cdot 2 \\
58 \cdot 1\end{array}$ & $\begin{array}{l}-\overline{34 \cdot 3} \\
34 \cdot 1 \\
31 \cdot 7\end{array}$ & $\begin{array}{r}\overline{3 \cdot 9} \\
6 \cdot 7 \\
10 \cdot 2\end{array}$ & $\begin{array}{l}-\overline{38 \cdot 6} \\
40 \cdot 8 \\
41.9\end{array}$ & $\begin{array}{l}37 \cdot 2 \\
35 \cdot 3 \\
28 \cdot 8 \\
31 \cdot 2\end{array}$ \\
\hline 452 & - & $\begin{array}{r}0 \\
2 \\
7 \\
40\end{array}$ & $\begin{array}{l}731 \\
697 \\
741 \\
898\end{array}$ & $\begin{array}{l}297 \\
286 \\
309 \\
329\end{array}$ & $\begin{array}{l}\mathbf{4 3 4} \\
\mathbf{4 1 1} \\
\mathbf{4 3 2} \\
\mathbf{5 6 9}\end{array}$ & $\begin{array}{l}\text { colost } \\
=\end{array}$ & $\bar{z}$ & $\bar{z}$ & $\bar{z}$ & $\bar{z}$ & $\begin{array}{l}41 \cdot 4 \\
41 \cdot 7 \\
42 \cdot 3 \\
37 \cdot 2\end{array}$ \\
\hline 415 & - & $\begin{array}{r}0 \\
3 \\
10 \\
36\end{array}$ & $\begin{array}{l}783 \\
805 \\
745 \\
902\end{array}$ & $\begin{array}{l}265 \\
255 \\
242 \\
344\end{array}$ & $\begin{array}{l}\mathbf{5 1 8} \\
\mathbf{5 5 0} \\
\mathbf{5 0 3} \\
\mathbf{5 5 8}\end{array}$ & $\begin{array}{l}<25 \\
<25 \\
<25 \\
<25\end{array}$ & $\bar{z}$ & $\bar{z}$ & $\bar{z}$ & $\bar{z}$ & $\begin{array}{l}34 \cdot 5 \\
32 \cdot 4 \\
33 \cdot 2 \\
38 \cdot 8\end{array}$ \\
\hline
\end{tabular}


Lamb no.

512

680

519

677

Colostrum given At birth

$12 \mathrm{hr}$.

$48 \mathrm{hr}$.

3 days

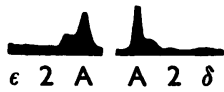

At birth

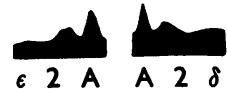

At birth

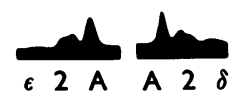

2 days

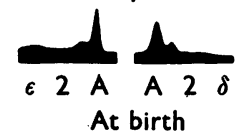

At birth

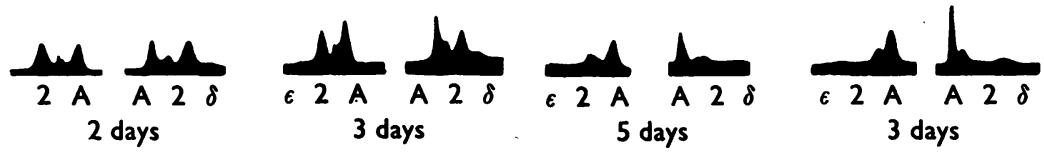

Age

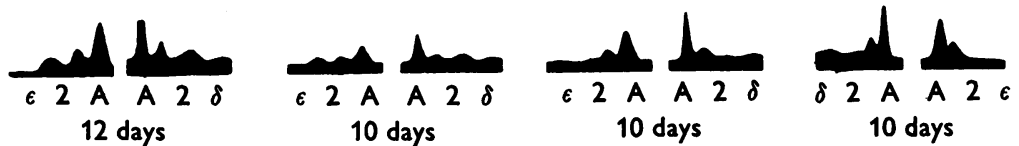

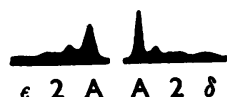

33 days

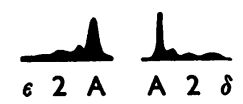
33 days

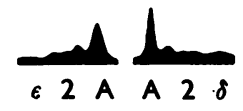

36 days

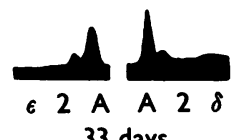

33 days

Fig. 1. Electrophoretic patterns of lambs' sera in phosphate buffer at pH 8.0 and ionic strength 0.2. The lambs were given colostrum after various periods of delay and bled at different ages.

Table 4. Examination of lambs' urine

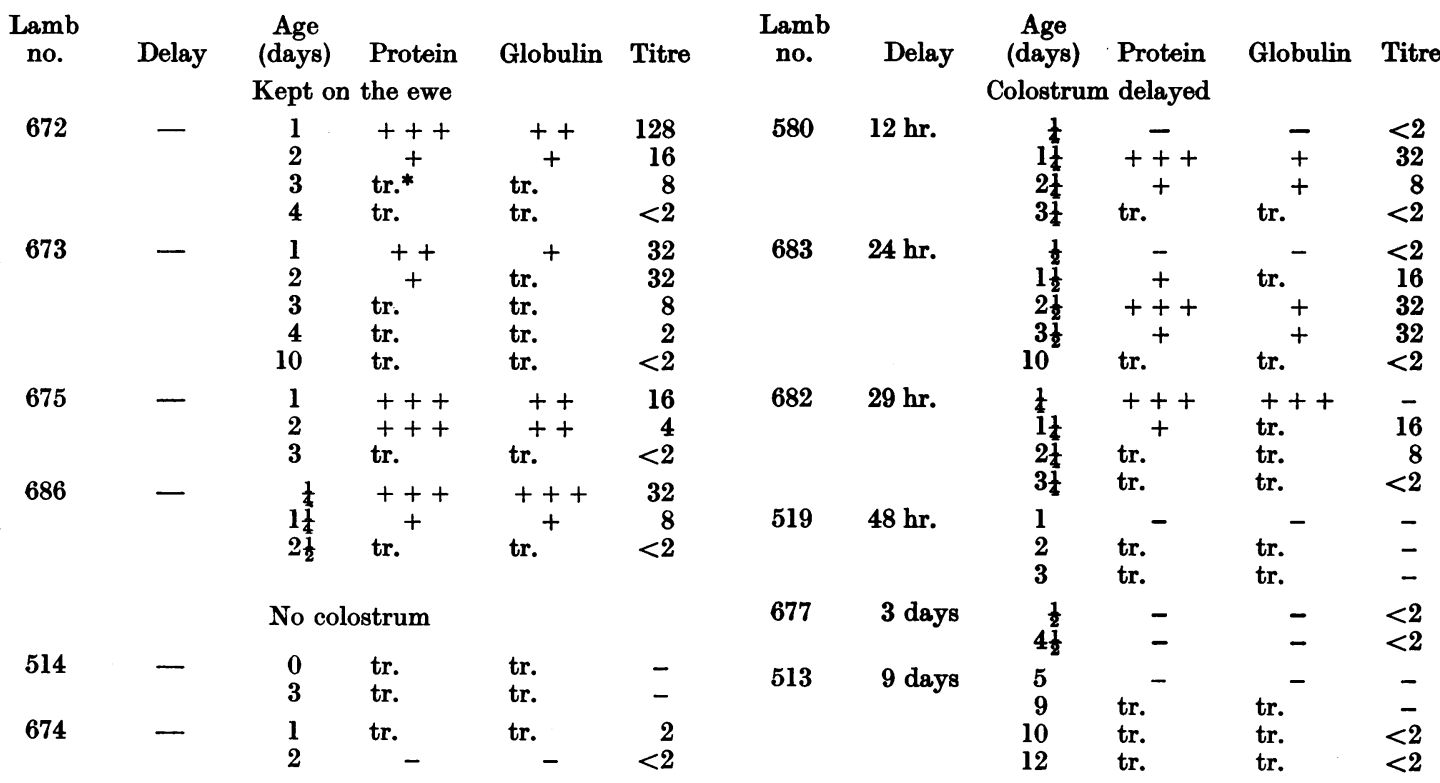

* tr. $=$ trace. 
are by no means in good or regular agreement. In only eleven out of twenty-eight cases the agreement was within $\pm 10 \%$. This may be explained in part by an observation of Jager, Schwartz, Smith, Nicherson \& Brown (1950) that too vigorous shaking of serum saturated with magnesium sulphate before filtration may seriously affect the results by protein denaturation.

Results on lambs' urine. These are reported in Table 4 and appear to provide a convenient index of the time of ingestion of colostrum. Proteinuria was marked when ingestion took place at birth and up to $29 \mathrm{hr}$. after birth; proteinuria was absent or only slight when colostrum was received after $48 \mathrm{hr}$. or not at all. The proteinuria appeared to include globulin and to be accompanied by an appreciable agglutinin titre. These effects became negligible in a few days.

\section{DISCUSSION}

The experimental data suggest that the changes observed in the lamb serum indicate the absorption of immune globulin from colostrum. These changes, together with those in the urine, show that ingestion of colostrum by the lambs up to some time between 29 and $48 \mathrm{hr}$. after birth leads to the absorption of immune globulin and the appearance or increase of a slow-moving electrophoretic component referred to as component 2. This finding may be compared with that obtained by Hansen \& Phillips (1947) on the calf. Their electrophoretic studies showed absorption of gamma globulin at 24 but not at $42 \mathrm{hr}$. after birth.

The urine tests showed that the kidney of the newborn lamb eppears to possess an unusual permeability, which persists for at least the same period after birth as that observed in the alimentary canal.

The permeability or otherwise of the different types of placenta has usually been explained in terms of the number of layers of tissue concerned. The present results indicate that this may not be so important as the properties of a particular layer since the impermeability of the placenta is in marked contrast to the permeability of the numerous tissue layers of the udder, alimentary canal and kidney. The results also show that the placental impermeability is not absolute, as sometimes a small amount of component 2 and a discernible agglutinin reaction could be obtained in the lambs' serum at birth (Table 3).

\section{SUMMARY}

1. Immunological, salt-precipitation and electrophoretic methods of investigation have been combined in a study of $(a)$ the changes in serum proteins of lambs on ingestion of colostrum at birth, $(b)$ the effect of delaying the ingestion of colostrum on these changes and (c) the occurrence of any proteinuria in this connexion.

2. The results show that there is an increase in serum globulin largely due to one electrophoretic component and an appearance of antibody, when colostrum is given up to $29 \mathrm{hr}$. but not at $48 \mathrm{hr}$. or more after birth.

3. Following the ingestion of colostrum the lambs showed a marked proteinuria, characterized by the presence of globulin and antibody.

We wish to acknowledge our indebtedness to $\mathrm{Mr}$ G. Anderson for technical assistance, to $\mathrm{Mr} \mathrm{W}$. Thomson and staff for management of the ewes and lambs and Mr R. Cook for the determination of agglutinin titres.

\section{REFERENCES}

Charlwood, P. A. \& Thomson, A. (1948). Nature, Lond., 61, 59.

Dalgarno, A., Godden, W. \& McCarthy, E. F. (1950). Biochem. J. 46, 162.

Earle, I. P. (1935). J. agric. Res. 51, 479.

Hansen, R. G. \& Phillips, P. H.(1947). J.biol.Chem.171, 223.

Jager, B. V., Schwartz, T. B., Smith, E. L., Nicherson, M. \&

Brown, D. M. (1950). J. Lab. clin. Med. 35, 76.
Mason, J. H., Dalling, T. \& Gordon, N. S. (1930). J. Path. Bact. 33, 783.

Orcutt, M. L. \& Howe, P. E. (1922). J. exp. Med. 36, 291.

Schnieder, L. \& Szathmary, J. (1939). Z. ImmunForsch. 95, 169.

Smith, T. \& Little, R. B. (1922). J. exp. Med. 36, 181. 